\title{
RITMOS DE ACTIVIDAD LOCOMOTORA Y TEMPERATURA EN Felis catus ${ }^{1}$
}

\author{
SciabarRasi, C. 2; Scaglione, M. C. ${ }^{2} ;$ Marozzi, M. ${ }^{2}$; \\ Elizalde, E. F. ${ }^{2}$ \& Cerutti, R. D. ${ }^{1}$
}

\begin{abstract}
RESUMEN
En este trabajo se analizan los ritmos de actividad locomotora y temperatura del gato doméstico (Felis catus). Se realizaron dos experimentos con 4 animales alojados en jaulas individuales. Cada animal portaba un actímetro y un thermocron. En el primer experimento se caracterizó la ritmicidad diaria de actividad y temperatura periférica bajo condiciones LO 12:12. El segundo experimento fue diseñado para estudiar el marcapaso endógeno mediante actimetría, se invirtió el ciclo LO y posteriormente se observó bajo condiciones de curso libre en OO, LL y pulsos ultradianos. Nuestros resultados muestran que el gato presenta actividad crepuscular con mayor actividad nocturna, los ritmos de actividad diaria se resincronizan rápidamente tras invertir el ciclo LO y persisten bajo condiciones de curso libre LL, lo que sugiere un origen circadiano. El incremento de temperatura periférica va asociado a una mayor propensión al reposo, mientras que los descensos inducen estados de vigilia.

Palabras claves: cronobiología, ritmos biológicos, fotoperiodo, actividad locomotora, temperatura periférica, Felis catus.
\end{abstract}

\section{SUMMARY}

\section{Rhythms of the locomotor activity and temperature in Felis catus.}

This work analyses the rhythms of the locomotor activity and temperature in domestic cats (Felis catus). Two experiments were carried out with four animals kept in individual cages. An actimeter and a temperature data logger were attached to each animal. In the first experiment the daily rhythmicity of activity and temperature under conditions LD 12:12 was characterized. In order to study the endogenous pacemaker by actigraphy, in the second experiment the LD cycle was inverted and then were studied under free course conditions in DD, LL and ultradian rhythms. Our results show that the cats present twilight activity with a higher nocturnal activity, the rhythms of daily activity

1.- C Subsidiado por UNL. Proyecto CAI+D 2013.

2.- Facultad de Ciencias Veterinarias (UNL). Kreder 2805. (3080) Esperanza, provincia de Santa Fe. Email: rcerutti@fcv.unl.edu.ar

Manuscrito recibido el 4 de septiembre de 2014 y aceptado para su publicación el 20 de enero de 2015. 
are quickly resynchronized after inverting the LD cycle and they persist under free course conditions LL, which suggests a circadian origin. The increase in temperature is associated with a higher propensity to rest, while declines induce states of wakefulness.

Key words: chronobiology, biological rhythms, photoperiod, locomotor activity, peripheral temperature, Felis catus.

\section{INTRODUCCIÓN}

A lo largo de la evolución los seres vivos han desarrollado relojes biológicos que se sincronizan con los cambios cíclicos que se dan en el medio ambiente como consecuencia de los ciclos geofísicos que originan la alternancia día-noche, las fases lunares y las estaciones. Los marcapasos internos tienen un papel fundamental en el control de los ritmos biológicos ya que permiten a los organismos medir el paso del tiempo, integrando la información ambiental recibida a través del órgano pineal y transducida por la hormona melatonina, a fin de anticipar eventos predecibles (p.ej. el atardecer o la primavera) y de este modo poder organizar recursos y actividades antes de que sean necesarios (Pitterndrigh, 1960).

La ritmicidad de la temperatura corporal y la actividad motora, son dos variables frecuentemente utilizadas en cronobiología para determinar la función del sistema circadiano (Hofstra \& Weerd, 2008). La estrecha relación entre el ciclo de sueño vigilia y el ritmo de temperatura corporal es un hecho que se conoce desde hace tiempo, de tal forma que todas las especies, con independencia de su carácter diurno o nocturno, duermen durante el periodo coincidente con el mínimo de temperatura corporal central (TCC) (Zulley et al., 1981). No obstante debido a los inconvenientes metodológicos que plantea la medida de TCC de forma continua, recientemente se ha propuesto el ritmo de temperatura periférica (TP) como ritmo marcador ya que presenta evidentes ventajas en su medida y es igualmente indicador del estado del sistema circadiano (Sarabia et al., 2008). Este ritmo es el resultado de la actividad del NSQ sobre el balance entre activación simpática (vasoconstricción y reducción de temperatura) y parasimpática (vasodilatación y aumento de temperatura) de los vasos sanguíneos de la piel. El comportamiento de TP en relación con el sueño es opuesto al del ritmo de TCC, de modo que la temperatura de la piel aumenta inmediatamente antes y durante los periodos de sueño y somnolencia y desciende durante los periodos de actividad en proporción al nivel de activación o arousal (Bonmati-Carrion et al., 2013).

Las oscilaciones en el comportamiento de la actividad se utilizan ampliamente como un indicador para evaluar el bienestar de los animales (Gordon, 1995). Se manifiesta en muchas especies (Enrigth, 1980) y han sido extensamente estudiada en humanos, animales de laboratorio, incluyendo ratas de laboratorio, ratones domésticos, hamsters, ardillas, degus, ratas topo, topillos, cobayas y también en conejos, perros, ovejas, cabras y caballos (Refinetti, 2006; Bertolucci et al., 2008; Piccione et al, 2007, 2008). En la literatura científica existen sólo unos pocos 